\title{
EDITORIAL
}

\section{Combating the cons of consolidation}

\author{
With another wave of major mergers and acquisitions imminent in 2009, protecting and \\ stimulating a culture of innovation will be crucial in tackling the RED productivity shortfall \\ that underlies ongoing industry consolidation.
}

\begin{abstract}
"A quantitative analysis of the drug discovery potential of the pharmaceutical industry leads to the conclusion that the industry is facing an innovation deficit which will be severe enough to incite further consolidations within the industry." This opening sentence, from a 1996 article $^{1}$ by Jürgen Drews - president of research at Roche at the time - and Stefan Ryser, is again, regrettably, seeming highly prescient. At the end of January, it was announced that Pfizer will acquire Wyeth for $\sim$ US $\$ 68$ billion, consolidating Pfizer's position as the largest pharmaceutical company for the next few years.
\end{abstract}

The rationale from Pfizer's perspective seems clear. Perhaps most significantly, in the next 2 years, it is due to face generic competition to its best-selling drug Lipitor, which had reported global sales of $\sim \$ 12$ billion in 2008 , representing more than a quarter of Pfizer's total prescription pharmaceutical sales. With no prospect of replacing the resultant loss of revenue with sales from new products, analysts' forecasts indicated that Pfizer would plummet from its current industry-leading position in terms of sales, providing a strong drive to purchase other large companies to stem the loss.

Wyeth is also facing problems with upcoming patent expiries, with generic competition to two of its blockbusters - Effexor and Protonix - expected in 2010-2011. Nevertheless, although projections indicate that the prescription pharmaceutical sales of a merged PfizerWyeth would be smaller in five years' time than those of the two companies combined at present, the merged company would still hold the top sales spot.

Looking beyond the volume of sales reveals other key factors for Pfizer. First, the acquisition will provide a boost to Pfizer's recent efforts to increase its presence in biologics and vaccine development; Wyeth products include the current top-selling biologic Enbrel and the top-selling vaccine Prevnar, and it has built up a strong biomanufacturing capability. As well as reducing Pfizer's dependence on small molecules, which are more susceptible to generic competition than biologics, the overall business will now have more of an emphasis on specialty pharmaceuticals, which are increasingly being pursued by pharmaceutical companies in general (see page 184). Second, the sheer size of the merged company, as well as its greater product diversification, might provide future insulation against sharp losses in revenue, as exemplified by the Lipitor patent expiry.

Ultimately, however, the key to future success is likely to be how well the company addresses the longacknowledged challenges of R\&D productivity. In this respect, some would argue that Pfizer's previous major acquisitions - Warner Lambert in 2000 and Pharmacia in 2003 - have together provided a strong example of how damaging mega-mergers can be. Indeed, since 2003, Pfizer has had just five new molecular entities approved by the US FDA.

Pfizer CEO Jeffrey Kindler has emphasized that the Wyeth merger will be very different from those preceding it, which were characterized by the acquisition of products such as Lipitor and dramatic cost-cutting. Nevertheless, major cost cuts are still anticipated, with estimates that $\$ 4$ billion could be saved by 2012, in part by reducing the combined workforce of the company by $\sim 15 \%$.

So, arguably the key challenge for Pfizer - as for other companies involved in consolidation - will be finding a way to ensure that the morale and innovative potential of researchers and teams are not destroyed in the process. The importance here is illustrated by the history ${ }^{2}$ of the blockbuster anticancer therapy Avastin, which is one of the drivers behind the other major deal anticipated at present: Roche's bid to acquire the whole of Genentech. This drug was based on the discovery of vascular endothelial growth factor by a small team at Genentech in the late 1980s, catalysed by the curiosity and perseverance of a single scientist. The value of small, highly motivated and creative research teams has increasingly been acknowledged by some of the companies created by the last wave of mega-mergers. It is to be hoped that their efforts, and those in general to create research cultures that allow such teams to thrive, might finally help address the innovation deficit in the pharmaceutical industry.

\footnotetext{
Drews, J. \& Ryser, S. Innovation deficit in the pharmaceutical industry. Drug Inf. J. 30, 97-107 (1996).

2. Ferrara, N. VEGF and the quest for tumour angiogenesis factors. Nature Rev. Cancer 2, 795-803 (2002).
} 